ARTICLE

\title{
Manipulation of hot carrier cooling dynamics in two-dimensional Dion-Jacobson hybrid perovskites via Rashba band splitting
}

\author{
Jun Yin (10) 1,2,6, Rounak Naphade 2,6, Partha Maity (1) 1,2,6, Luis Gutiérrez-Arzaluz (1) 1,2, Dhaifallah Almalawi ${ }^{3,4}$, \\ Iman S. Roqan (1) ${ }^{3}$, Jean-Luc Brédas (1) ${ }^{5 凶}$, Osman M. Bakr (D) ${ }^{2 \otimes} \&$ Omar F. Mohammed (1) ${ }^{1,2 \bowtie}$
}

Hot-carrier cooling processes of perovskite materials are typically described by a single parabolic band model that includes the effects of carrier-phonon scattering, hot phonon bottleneck, and Auger heating. However, little is known (if anything) about the cooling processes in which the spin-degenerate parabolic band splits into two spin-polarized bands, i.e., the Rashba band splitting effect. Here, we investigated the hot-carrier cooling processes for two slightly different compositions of two-dimensional Dion-Jacobson hybrid perovskites, namely, (3AMP) $\mathrm{Pbl}_{4}$ and (4AMP)Pbl 4 (3AMP = 3-(aminomethyl)piperidinium; $4 \mathrm{AMP}=4$ (aminomethyl)piperidinium), using a combination of ultrafast transient absorption spectroscopy and first-principles calculations. In (4AMP)Pbl 4 , upon Rashba band splitting, the spindependent scattering of hot electrons is responsible for accelerating hot-carrier cooling at longer delays. Importantly, the hot-carrier cooling of (4AMP) $\mathrm{Pbl}_{4}$ can be extended by manipulating the spin state of the hot carriers. Our findings suggest a new approach for prolonging hot-carrier cooling in hybrid perovskites, which is conducive to further improving the performance of hot-carrier-based optoelectronic and spintronic devices.

\footnotetext{
${ }^{1}$ Advanced Membranes and Porous Materials Center, Division of Physical Science and Engineering, King Abdullah University of Science and Technology, Thuwal 23955-6900, Kingdom of Saudi Arabia. ${ }^{2}$ KAUST Catalysis Center, Division of Physical Sciences and Engineering, King Abdullah University of Science and Technology, Thuwal 23955-6900, Kingdom of Saudi Arabia. ${ }^{3}$ Division of Physical Sciences and Engineering, King Abdullah University of Science and Technology, Thuwal 23955-6900, Kingdom of Saudi Arabia. ${ }^{4}$ Department of Physics, College of science, Taif University, Taif 21944, Saudi Arabia.

${ }^{5}$ Department of Chemistry and Biochemistry, The University of Arizona, Tucson 85721-0088 AZ, USA. ${ }^{6}$ These authors contributed equally: Jun Yin, Rounak Naphade, Partha Maity.『email: jlbredas@arizona.edu; osman.bakr@kaust.edu.sa; omar.abdelsaboor@kaust.edu.sa
} 
T wo-dimensional (2D) hybrid perovskites are promising optoelectronic semiconductors because they offer greater structural diversity and stability compared to their threedimensional (3D) counterparts ${ }^{1-3}$. In addition, the structural arrangement in $2 \mathrm{D}$ hybrid perovskites is akin to quantum wells (i.e., inorganic layers electronically isolated by insulating organic layers), giving rise to intriguing physical phenomena, such as quantum and dielectric confinement effects ${ }^{4}$, the optical Stark effect ${ }^{5}$, and coherent phonon interactions $s^{6-8}$. In addition, similar to 3D hybrid perovskites, the presence of heavy atoms (e.g., lead) can induce Rashba band splitting because of inversion symmetry breaking ${ }^{9,10}$, making such materials promising candidates for manipulating the spin states in spintronic devices. On the other hand, the spontaneous polarization properties of ferroelectric $2 \mathrm{D}$ perovskites can enhance free charge carrier generation and break the Shockley-Queisser limit for the bulk photovoltaic effect ${ }^{11,12}$.

There is also a growing interest in exploring applications involving hot-carrier-based optoelectronic and spintronic devices, especially hot-carrier solar cells (HCSCs), using 2D hybrid perovskites due to the deceleration of their hot-carrier cooling rate, which is mainly governed by quantum and dielectric confinement ${ }^{8,13,14}$. In this case, the hot-carrier cooling time can be extended up to tens of picoseconds via the combined effects of hot-phonon bottleneck ${ }^{15}$, enhanced Auger heating ${ }^{16}$, and formation of large polarons ${ }^{17-19}$. The cooling processes of hot carriers in perovskite materials are typically described using the single parabolic band model by assuming that the photogenerated hot carriers experience cooling via carrier-phonon scattering, optical phonon emission, acoustic-optical phonon upconversion, and thermal equilibrium ${ }^{16,20}$. However, when the spin degeneracy of the parabolic band is lifted and two spin-polarized band form, the cooling processes will be strongly influenced by having hot carriers with different spin states. Thus, understanding the mechanisms behind the hot-carrier dynamics in $2 \mathrm{D}$ perovskite systems upon Rashba band splitting is critical to realizing their applications in new optoelectronic and spintronic devices.

For typical single-layered (001)-oriented 2D hybrid perovskites $(n=1)$ with the general formula $\mathrm{A}_{2} \mathrm{MX}_{4}$ or $\mathrm{AMX}_{4}(\mathrm{~A}=$ organic cation; $\mathrm{M}=\mathrm{Pb}$ or $\mathrm{Sn} ; \mathrm{X}=\mathrm{Cl}^{-}, \mathrm{Br}^{-}$, or $\mathrm{I}^{-}$), the structural, electronic, and photophysical properties are strongly dependent on the nature of the organic spacers (e.g., size, shape, and electron affinity), as well as the configuration of the inorganic layers. Compared to the extensively studied 2D Ruddlesden-Popper (RP) perovskites with monovalent cations, the 2D Dion-Jacobson (DJ) perovskites show shorter interlayer distances and better stability because diammonium cations $(+2)$ can strongly interact with the inorganic layers via both hydrogen bonding and interlayer van der Waals I ...I interactions ${ }^{21,22}$. Herein, we explored and deciphered the hot-carrier cooling processes in two slightly different compositions of 2D DJ perovskites, namely, (3AMP) $\mathrm{PbI}_{4}$ and $\quad(4 \mathrm{AMP}) \mathrm{PbI}_{4} \quad(3 \mathrm{AMP}=3$-(aminomethyl)piperidinium; $4 \mathrm{AMP}=4$-(aminomethyl)piperidinium), by combining ultrafast time-resolved spectroscopy and first-principles calculations. In particular, we seek to understand the effect of Rashba band splitting on the cooling processes. We first confirmed the Rashba band splitting in (4AMP) $\mathrm{PbI}_{4}$ by a combination of electronic

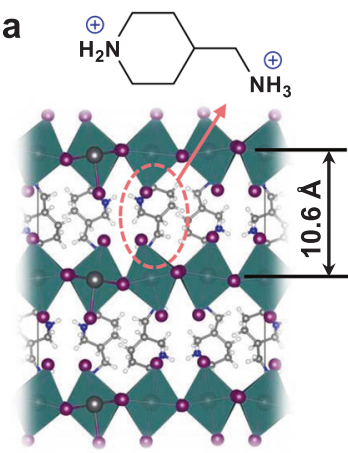

(3AMP)Pbl

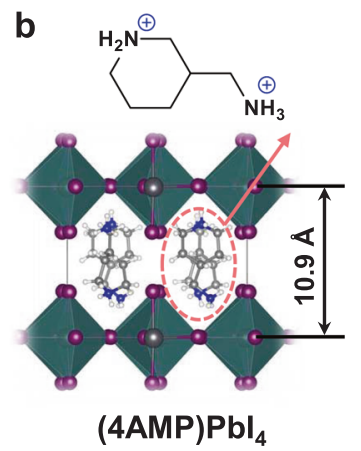

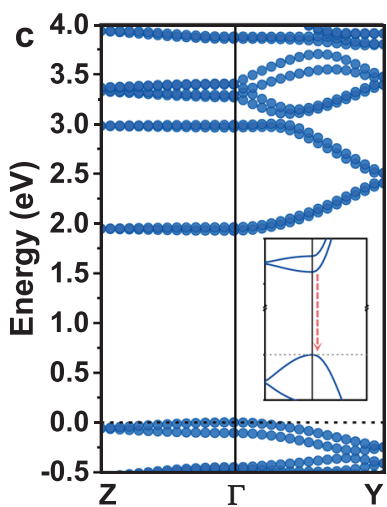
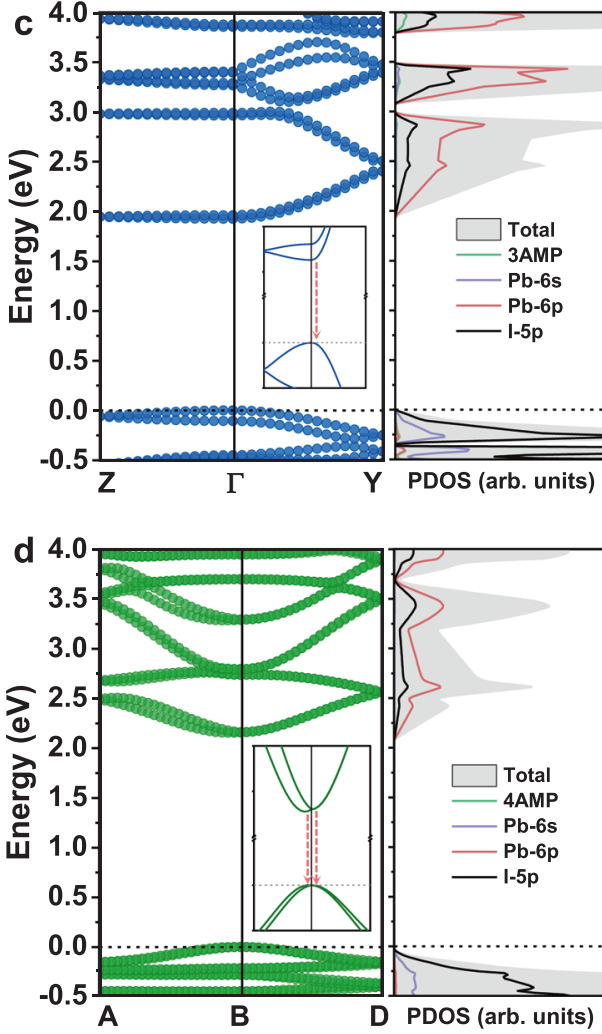

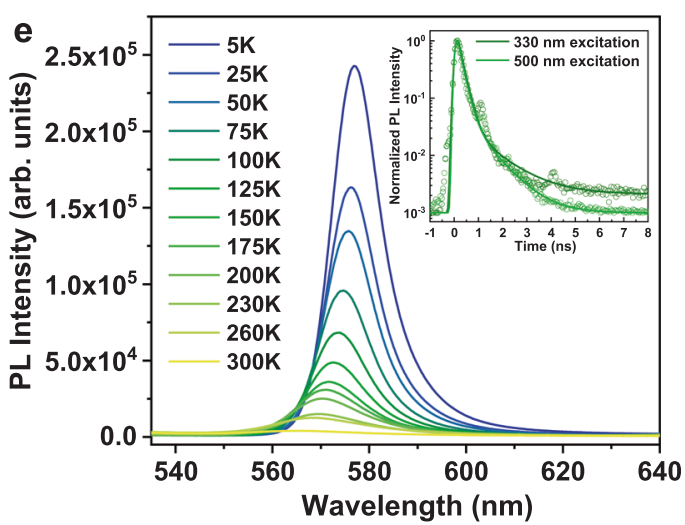

$f$

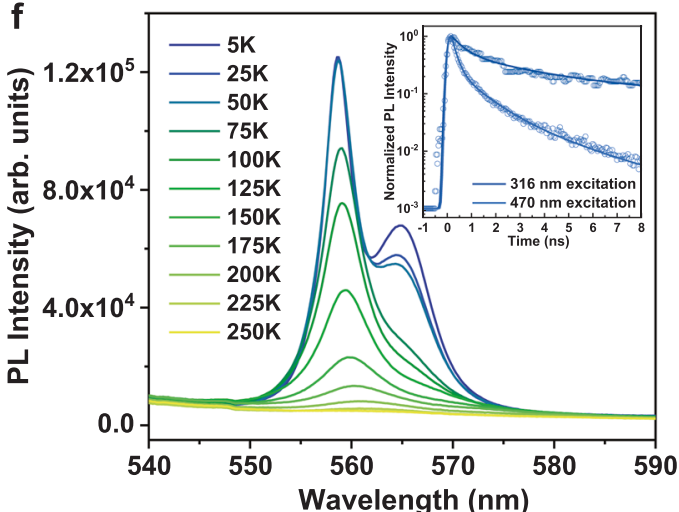

Fig. 1 Crystal structure, electronic bands, and PL spectra of 2D Dion-Jacobson perovskites. $\mathbf{a}$, b Crystal structures of $2 \mathrm{D}$ Dion-Jacobson perovskites, (3AMP) $\mathrm{Pbl}_{4}$ and (4AMP)Pbl 4 , together with the chemical structures of the organic cations. c, d Electronic bands and total and projected density of states (PDOS) of (3AMP)Pbl 4 and (4AMP)Pbl 4 calculated at the DFT HSEO6 level with spin-orbit coupling (SOC). Enlarged electronic bands near the valence band maximum and conduction band minimum are shown in the insets. e, $\mathbf{f}$ Temperature-dependent PL spectra of the ( $3 \mathrm{AMP}$ )Pbl ${ }_{4}$ and (4AMP)Pbl ${ }_{4}$ films, together with the time-resolved PL decays for the emission from the ( $3 \mathrm{AMP}) \mathrm{Pbl}_{4}$ film at $565 \mathrm{~nm}$ and the emission from the (4AMP)Pbl ${ }_{4}$ film measured at $560 \mathrm{~nm}$ and room temperature. The solid lines represent fits to a biexponential decay function. Note that the observed spikes in (e) could be due to the presence of film irregularities that lead to small reflections inside the film. 
band structure calculations and temperature-dependent photoluminescence $(\mathrm{PL})$ and time-resolved PL experiments. In both (3AMP) $\mathrm{PbI}_{4}$ and (4AMP) $\mathrm{PbI}_{4}$, the carrier-hot phonon interactions combined with the hot-phonon effect are responsible for the fast relaxation of hot carriers at early times (less than $1 \mathrm{ps}$ ). The nonadiabatic molecular dynamics (NAMD) simulations reveal that the fast intraband relaxations to the band edges are governed by hybrid vibrations and nonadiabatic couplings between the initial and lower states. Moreover, we attribute the fast hot-carrier cooling at longer time delays (up to hundreds of picoseconds) to spin-flip/precession and spin-phonon scattering of hot electrons. In addition, by controlling the spin state of the hot carriers generated upon circular copolarized excitation, we observe slower hot-carrier cooling in (4AMP) $\mathrm{PbI}_{4}$ that displays Rashba band splitting. This is also supported by NAMD calculations considering the spin-orbit coupling and decoherence effects.

\section{Results and Discussion}

Structural, electronic bands, and optical properties of 2D DJ perovskites. The $2 \mathrm{D}$ Dion-Jacobson perovskite (3AMP) $\mathrm{PbI}_{4}$ and (4AMP) $\mathrm{PbI}_{4}$ films were prepared from their single crystals using a previously reported method ${ }^{23,24}$ with some modifications (see Materials and Methods for details). The X-ray diffraction (XRD) patterns validate the formation of the $2 \mathrm{D}$ phase and the compositional purity (Supplementary Fig. 1a). It also confirms that the perovskite layers are stacked perpendicular to the substrate plane. Atomic force microscopy (AFM) images show a smooth topography of microcrystalline domains that are merged to form compact films (Supplementary Fig. 2). For comparison purposes, 2D Ruddlesden-Popper hybrid perovskites, $(\mathrm{PMA})_{2} \mathrm{PbI}_{4}$ and $(\mathrm{PEA})_{2} \mathrm{PbI}_{4}$, were also investigated here. From the crystal structures of (3AMP) $\mathrm{PbI}_{4}$ and (4AMP) $\mathrm{PbI}_{4}$ (Fig. 1a, b), we find the following: (i) $2 \mathrm{D}$ DJ perovskites contain one sheet of divalent +2 cations between the inorganic layers, in which the cations have a balanced positive charge density for both $\mathrm{C}_{5} \mathrm{NH}_{11}+$ and $\mathrm{NH}_{3}+$ (see the electrostatic potential surfaces in Supplementary Fig. 3). (ii) $\mathrm{The} \mathrm{Pb}-\mathrm{I}-\mathrm{Pb}$ angles in $2 \mathrm{D} \mathrm{DJ}$ perovskites are much more distorted as they are directly exposed to the organic cations. (iii) The strong hydrogen bonding between the organic cations and the inorganic layers can prevent rotational motion, resulting in more restricted cations or fewer degrees of freedom and short interlayer distances of $10.6 \AA$ and $10.9 \AA$ for $(3 \mathrm{AMP}) \mathrm{PbI}_{4}$ and (4AMP) $\mathrm{PbI}_{4}$, respectively. In contrast, the $2 \mathrm{D} \mathrm{RP}$ perovskites, $(\mathrm{PMA})_{2} \mathrm{PbI}_{4}$ and $(\mathrm{PEA})_{2} \mathrm{PbI}_{4}$, contain two sheets of interdigitating monovalent +1 cations with a positive charge density for $\mathrm{NH}_{3}{ }^{+}$, leading to more flexible layer stacking and larger distances between the inorganic layers: $15.1 \AA$ for $(\mathrm{PMA})_{2} \mathrm{PbI}_{4}$ and $16.5 \AA$ for $(\mathrm{PEA})_{2} \mathrm{PbI}_{4}$ (the crystal structures are shown in Supplementary Fig. 4).

The optical band gaps of the (3AMP) $\mathrm{PbI}_{4}$ and (4AMP) $\mathrm{PbI}_{4}$ films were deduced from the onsets of the absorption spectra to be $2.16 \mathrm{eV}$ and $2.32 \mathrm{eV}$, respectively (Supplementary Fig. 1b); these values are consistent with the direct band gaps calculated for (3AMP) $\mathrm{PbI}_{4}$ at the $\Gamma$-point (GGA/PBE: $2.08 \mathrm{eV}$; HSE + SOC: $1.93 \mathrm{eV}$ ) and (4AMP) $\mathrm{PbI}_{4}$ at the B-point (GGA/PBE: $2.22 \mathrm{eV}$; HSE + SOC: $2.16 \mathrm{eV}$ ), as shown in Fig. 1c, d. The complete electronic band structures calculated at the GGA/PBE with and without SOC are shown in Supplementary Figs. 5 and 6. Compared to (3AMP) $\mathrm{PbI}_{4}$, the better stacking of inorganic layers in (4AMP) $\mathrm{PbI}_{4}$ allows stronger interlayer electronic couplings via van der Waals I...I interactions, leading to reduced band gaps via enhanced antibonding interactions and destabilization of the valence bands. The corresponding projected densities of states (PDOSs) suggest that hybridization of $\mathrm{Pb}-6 \mathrm{~s}$ and I-5p orbitals mainly contributes to the valence bands, while the Pb-6p orbital has a dominant contribution to the conduction bands.

The (4AMP) $\mathrm{PbI}_{4}$ crystal belongs to the $\mathrm{P} c$ space group and the inorganic layers match perfectly with an averaged equatorial $\mathrm{Pb}-\mathrm{I}-\mathrm{Pb}$ angle of $155^{\circ}$. Such polar octahedral distortions can break the inversion symmetry, leading to Rashba band splitting around the conduction band edge (Fig. 1d). The Rashba splitting coefficient $\alpha_{\mathrm{R}}$ (defined as $2 E_{\mathrm{R}} / k_{0}$, where $k_{0}$ is the momentum offset and $E_{\mathrm{R}}$ is the energy splitting) for ( $\left.4 \mathrm{AMP}\right) \mathrm{PbI}_{4}$ is $1.46 \mathrm{eV} \cdot \AA$, which is on the same order of magnitude as that obtained from recent experiments $\left(\alpha_{\mathrm{R}}=2.6 \mathrm{eV} \cdot \AA\right)^{21}$. On the other hand, (3AMP) $\mathrm{PbI}_{4}$ belongs to the centrosymmetric $\mathrm{P} 21 / c$ space group; here, the inorganic layers stack on top of one another with a larger averaged equatorial $\mathrm{Pb}-\mathrm{I}-\mathrm{Pb}$ angle of $165^{\circ}$, and no band splitting is observed for either the conduction band or valence band edge. Such structural and electronic differences between (3AMP) $\mathrm{PbI}_{4}$ and (4AMP) $\mathrm{PbI}_{4}$ can be attributed to the stronger hydrogen bonding interactions between $\mathrm{H}$ atoms from $\mathrm{NH}_{3}{ }^{+}$and $\mathrm{I}$ atoms in (4AMP) $\mathrm{PbI}_{4}$ (see the comparison of overlap population for hydrogen bonding in Supplementary Table 1). It should be noted that band splitting is also absent in the 2D RP perovskites ( $\mathrm{PMA})_{2} \mathrm{PbI}_{4}$ and $(\mathrm{PEA})_{2} \mathrm{PbI}_{4}$ due to the absence of tilting of the $\mathrm{Pb}-\mathrm{I}-\mathrm{Pb}$ angles.

To confirm the Rashba band splitting in (4AMP) $\mathrm{PbI}_{4}$, we first carried out temperature-dependent $\mathrm{PL}$ measurements ranging from $5 \mathrm{~K}$ to $300 \mathrm{~K}$. As shown in Fig. 1e, (3AMP) $\mathrm{PbI}_{4}$ shows a single emission peak $(564.8 \mathrm{~nm})$ at room temperature and exhibits a spectral red-shift for decreasing temperature. In contrast, the emission peak for (4AMP) $\mathrm{PbI}_{4}$ starts to split into two for temperatures lower than $150 \mathrm{~K}$ (Fig. 1f); and two emission peaks located at $558.7 \mathrm{~nm}$ and $564.9 \mathrm{~nm}$ are observed at $5 \mathrm{~K}$, which can be attributed to the two split bands (see the insert of Fig. 1f). This also agrees well with recent observations of robust ferroelectricity and Rashba band splitting in (4AMP) $\mathrm{PbI}_{4}$ via circularly polarized PL measurements ${ }^{21}$. We further performed time-resolved PL (TRPL) measurements to confirm the band splitting in (4AMP) $\mathrm{PbI}_{4}$. For excitation close to the band edge, (3AMP) $\mathrm{PbI}_{4}$ shows an average PL lifetime of $0.17 \mathrm{~ns}$, which is slightly shorter than that obtained with high-energy excitation $\left(\tau_{\text {ave }}=0.27 \mathrm{~ns}\right)$. Similar PL decays are observed in 2D RP perovskites (Supplementary Fig. 7), showing an average PL lifetime of $0.25 \mathrm{~ns}$ and $0.34 \mathrm{~ns}$ for $\left(\mathrm{PMA}_{2} \mathrm{PbI}_{4}\right.$ and $(\mathrm{PEA})_{2} \mathrm{PbI}_{4}$, respectively. In contrast, $(4 \mathrm{AMP}) \mathrm{PbI}_{4}$ shows a much longer $\mathrm{PL}$ lifetime, especially with high-energy excitation ( $\tau_{\text {ave }}=7.69 \mathrm{~ns}$ ). Such slower radiative recombination in $(4 \mathrm{AMP}) \mathrm{PbI}_{4}$ can be attributed to the indirect bandgap nature induced by spinpolarized Rashba band splitting ${ }^{10,25}$.

Hot carrier cooling processes in 2D DJ perovskites. To assess the hot-carrier cooling properties in 2D DJ perovskite films following excitation at excess energy, we performed femtosecond transient absorption (fs-TA) spectroscopy at different excitation fluences. Figure $2 \mathrm{a}-\mathrm{d}$ show the normalized TA spectra of $2 \mathrm{D}$ DJ perovskite films at high-energy excitation, i.e., $330 \mathrm{~nm}(3.76 \mathrm{eV})$ for (3AMP) $\mathrm{PbI}_{4}$ and $316 \mathrm{~nm}(3.92 \mathrm{eV})$ for $(4 \mathrm{AMP}) \mathrm{PbI}_{4}$, with pump fluences of $2.0 \mu \mathrm{J} / \mathrm{cm}^{2}$ and $4.0 \mu \mathrm{J} / \mathrm{cm}^{2}$ to minimize Auger recombination (see Supplementary Note 1). TA spectra at a lower pump fluence of $0.8 \mu \mathrm{J} / \mathrm{cm}^{2}$ were also obtained and are shown in Supplementary Fig. 8 . Note that the carrier densities $\left(n_{0}\right)$ corresponding to pump fluences of $2.0 \mu \mathrm{J} / \mathrm{cm}^{2}$ and $4.0 \mu \mathrm{J} / \mathrm{cm}^{2}$ are $8.25 \times$ $10^{17}$ and $1.65 \times 10^{18} \mathrm{~cm}^{-3}$ for (3AMP) $\mathrm{PbI}_{4}$ and $8.80 \times 10^{17}$ and $1.76 \times 10^{18} \mathrm{~cm}^{-3}$ for $(4 \mathrm{AMP}) \mathrm{PbI}_{4}$ (see Supplementary Note 2). Upon photoexcitation, the TA spectra of both the (3AMP) $\mathrm{PbI}_{4}$ and the (4AMP) $\mathrm{PbI}_{4}$ films show photobleaching $(\mathrm{PB})$ peak $(-\Delta \mathrm{A}>0)$ with high-energy tails near the bandgap due to band filling effects, 

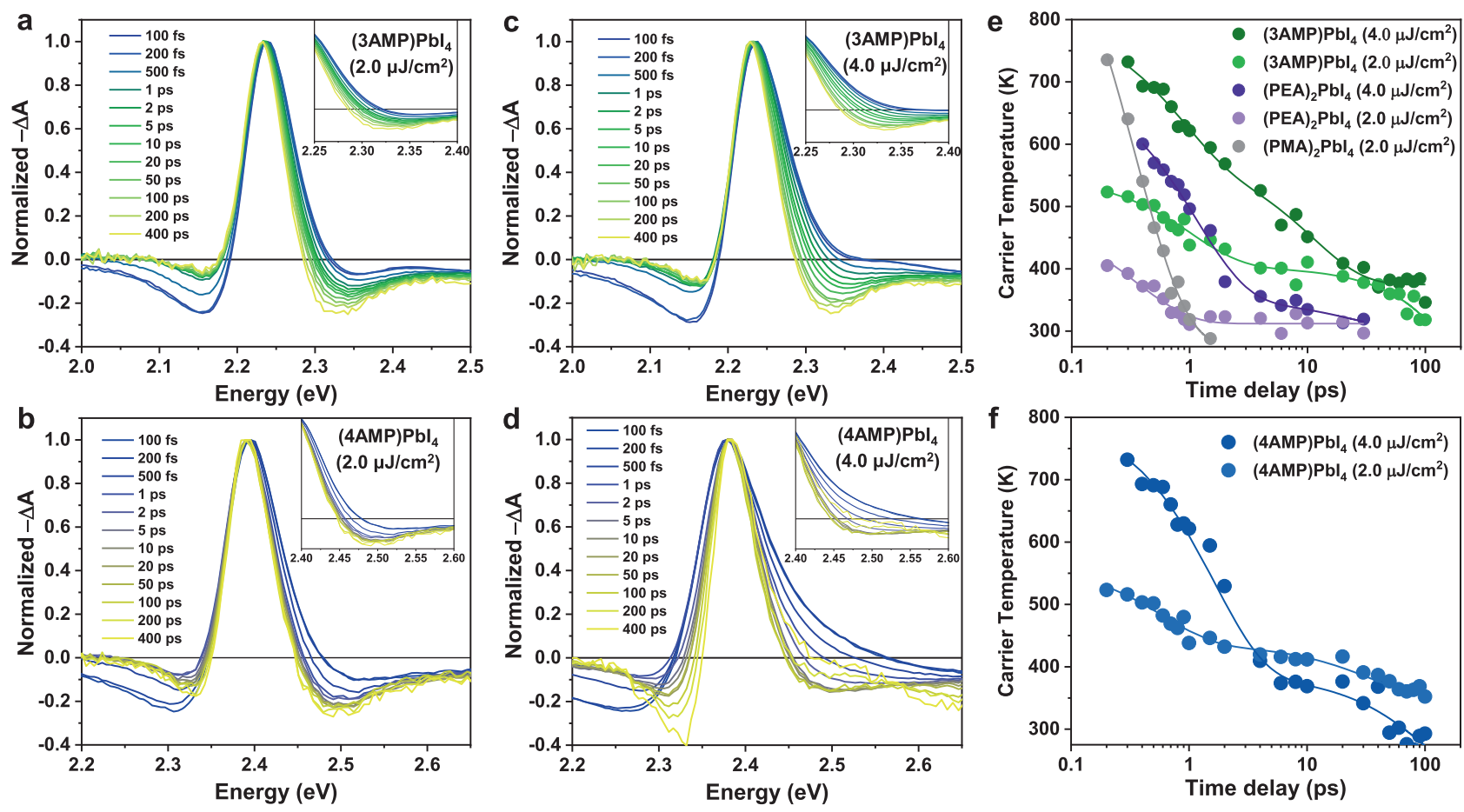

Fig. 2 Hot carrier cooling dynamics of 2D Dion-Jacobson perovskites. Normalized transient absorption spectra measured at an excitation wavelength of $(\mathbf{a}, \mathbf{c}) 330 \mathrm{~nm}$ for the (3AMP)Pbl film and (b, d) $316 \mathrm{~nm}$ for the (4AMP)Pbl 4 film with a pump fluence of $2.0 \mu \mathrm{J} / \mathrm{cm}^{2}$ and $4.0 \mu \mathrm{J} / \mathrm{cm}^{2}$. Extracted hot-carrier temperatures as a function of delay time for (e) (3AMP)Pbl $\mathrm{P}_{4}$ and (f) (4AMP)Pbl ${ }_{4}$ at different pump fluences $\left(2.0 \mu \mathrm{J} / \mathrm{cm}^{2} \mathrm{and} 4.0 \mu \mathrm{J} / \mathrm{cm}^{2}\right)$. The hot carrier temperatures of $(\mathrm{PMA})_{2} \mathrm{Pbl}_{4}$ and $(\mathrm{PEA})_{2} \mathrm{Pbl}_{4}$ are shown with gray and purple circles for comparison. The solid lines represent fits to a biexponential decay function.

as well as broad below-bandgap photoinduced absorption (PIA, $-\Delta \mathrm{A}<0)$ and above-bandgap PIA due to the change in the imaginary part of the refractive index ${ }^{26}$. Note that the band gap renormalization is almost negligible in both cases. With time, the high-energy tails gradually narrow, which suggests that the hotcarrier cooling process is accompanied by longitudinal optical (LO) phonon emission ${ }^{16}$.

The hot-carrier temperature $\left(T_{\mathrm{c}}\right)$ can be extracted by fitting the high-energy tails of the TA spectra using the Maxwell-Boltzmann function $\exp \left[\left(E_{\mathrm{f}}-E\right) / k_{\mathrm{B}} T_{\mathrm{c}}\right]$, where $k_{\mathrm{B}}$ is the Boltzmann constant and $E_{\mathrm{f}}$ is the quasi-Fermi energy (see Supplementary Note 3$)^{27}$. Figure $2 \mathrm{e}, \mathrm{f}$ show the $T_{\mathrm{c}}$ evolution following high-energy excitation at different pump fluences. At a low pump fluence of $2.0 \mu \mathrm{J} / \mathrm{cm}^{2}$, the initial $T_{\mathrm{c}}$ in the (3AMP) $\mathrm{PbI}_{4}$ film is $524 \mathrm{~K}$, and it cools fast with a time constant of $1.25 \mathrm{ps}$ (obtained from the biexponential fittings of initial $T_{\mathrm{c}}$ decays, see Supplementary Table 3) and an energy loss rate of $1.22 \mathrm{eV} / \mathrm{ps}$ (i.e., the initial excess energy divided by the cooling time) until $T_{\mathrm{c}}$ approaches room temperature. At a higher pump fluence of $4.0 \mu \mathrm{J} / \mathrm{cm}^{2}$, the initial $T_{\mathrm{c}}$ of the (3AMP)PbI ${ }_{4}$ film reaches $726 \mathrm{~K}$, and the hot electrons require a longer time to relax to the conduction band edge, giving a time constant of $2.46 \mathrm{ps}$ and an energy loss rate of $0.62 \mathrm{eV} / \mathrm{ps}$. Note that the $2 \mathrm{D} \mathrm{RP}$ perovskite $(\mathrm{PEA})_{2} \mathrm{PbI}_{4}$ film shows similar evolutions for the high-energy tails in the TA spectra (Supplementary Fig. 9) but exhibits much lower initial $T_{\mathrm{C}}$, as well as $T_{\mathrm{c}}$ time constant $\left(0.36 / 1.19 \mathrm{ps}\right.$ for $(\mathrm{PEA})_{2} \mathrm{PbI}_{4}$ at low/ high pump fluence), compared to that of (3AMP) $\mathrm{PbI}_{4}$. Although the initial $T_{\mathrm{c}}$ of the (PMA) ${ }_{2} \mathrm{PbI}_{4}$ film is even higher than that of (3AMP) $\mathrm{PbI}_{4}$ under the same pump fluence, $T_{\mathrm{c}}$ experiences a fast decay with a $T_{\mathrm{c}}$ time constant of $0.38 \mathrm{ps}$. It is worth mentioning that the $(\mathrm{PMA})_{2} \mathrm{PbI}_{4}$ film is not stable and may undergo a phase change under a higher pump fluence of $4.0 \mu \mathrm{J} / \mathrm{cm}^{2}$, as a new photobleaching signal appears at a wavelength of $408 \mathrm{~nm}$ (Supplementary Fig. 10). From these results, it can be concluded that the initial hot-carrier cooling in $2 \mathrm{D}$ hybrid perovskites is dominated within the subpicosecond time scale $(<1 \mathrm{ps})$ by the emission of optical phonons, while an additional slow cooling process is observed on a timescale of up to hundreds of picoseconds, especially at a high pump fluence, which can be attributed to the hot-phonon bottleneck effect ${ }^{15,26}$.

The (4AMP) $\mathrm{PbI}_{4}$ film also exhibits a high initial $T_{\mathrm{c}}$ (523 and 723 $\mathrm{K})$ and rapid initial cooling behavior during thermal equilibrium between the LO-phonon population and hot carriers $(<1 \mathrm{ps})$. The $T_{\mathrm{c}}$ time constant of (4AMP) $\mathrm{PbI}_{4}$ is $1.50 \mathrm{ps}$ at a low pump fluence (energy loss rate of $1.01 \mathrm{eV} / \mathrm{ps}$ ), and it becomes longer (2.37 ps) at a high pump fluence. The initial fast cooling in (4AMP) $\mathrm{PbI}_{4}$ is comparable to that of (3AMP) $\mathrm{PbI}_{4}$ and $2 \mathrm{D}$ RP perovskites, suggesting similar carrier-phonon interactions in $n=12 \mathrm{D}$ perovskites. Furthermore, compared to ( $3 \mathrm{AMP}) \mathrm{PbI}_{4}$, the lower density of states in the conduction bands for $(4 \mathrm{AMP}) \mathrm{PbI}_{4}$ (Fig. 1c, d) will lead to a reduced energy loss rate of hot carriers due to the presence of fewer available relaxation pathways. However, on a timescale of tens to hundreds of picoseconds, the high-energy tails of the photobleaching signals become fast evolving, indicating that an additional channel is involved in accelerating hot carrier cooling. This faster cooling of the hot carriers closer to the band edges, especially at high pump fluence, can be influenced by spin randomizations and flips of hot electrons from the split bands. Thus, we attribute the further fast $T_{\mathrm{c}}$ decays taking place on the timescale of tens to hundreds of picoseconds to the Rashba band splitting effect, which will be discussed in the following sections.

Intraband relaxations in 2D DJ perovskites. Figure $3 \mathrm{a}$, b show the normalized TA kinetics of the (3AMP) $\mathrm{PbI}_{4}$ and (4AMP) $\mathrm{PbI}_{4}$ films probed at the photobleaching peaks with different pump fluences. The buildup of band-edge bleach has been previously used to elucidate hot-carrier cooling processes ${ }^{28,29}$. However, 

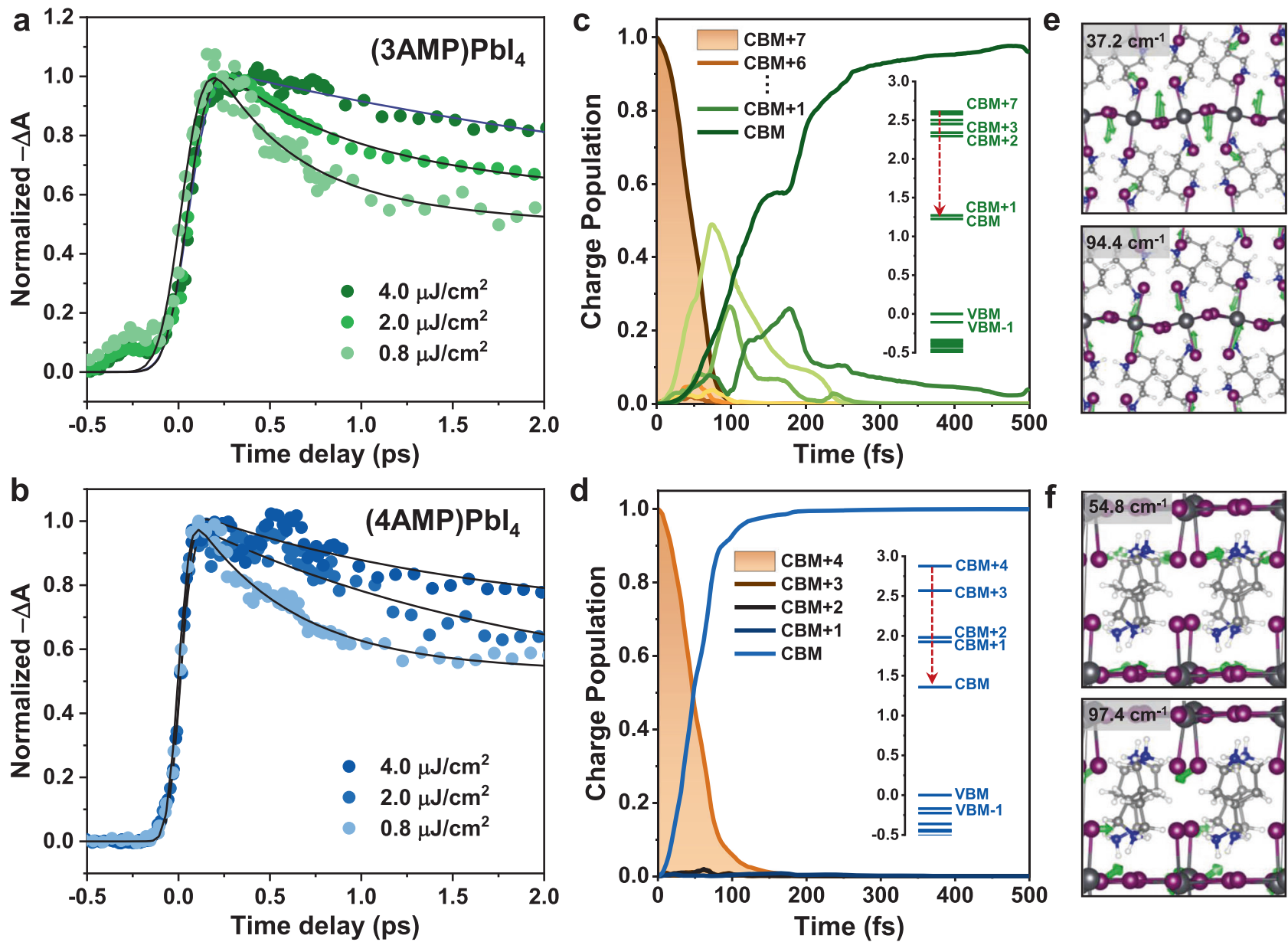

Fig. 3 Kinetics and intraband relaxation in 2D Dion-Jacobson perovskites. Normalized transient absorption kinetics (a) probed at a wavelength of 554 $\mathrm{nm}$ for the (3AMP)Pbl film (excitation at $330 \mathrm{~nm}$ ) and (b) probed at a wavelength of $518 \mathrm{~nm}$ for the (4AMP)Pbl 4 film (excitation at $316 \mathrm{~nm}$ ), with different pump fluences of $0.8 \mu \mathrm{J} / \mathrm{cm}^{2}, 2.0 \mu \mathrm{J} / \mathrm{cm}^{2}$, and $4.0 \mu \mathrm{J} / \mathrm{cm}^{2}$. The solid lines show best fits to the experimental data with an exponential function. Time evolution of hot electron relaxation (c) starting from CBM +7 for $(3 \mathrm{AMP}) \mathrm{Pbl}_{4}$ (excess energy $=1.52 \mathrm{eV}$ ) and $(\mathbf{d})$ starting from $\mathrm{CBM}+4$ for $(4 \mathrm{AMP})$ $\mathrm{Pbl}_{4}$ (excess energy $=1.38 \mathrm{eV}$ ). Inserts show the electronic energy levels of $(3 A M P) \mathrm{Pbl}_{4}$ and $(4 A M P) P b l_{4}$ at the high-symmetry $\Gamma$-point and $B$-point, respectively, involved in the nonadiabatic molecular dynamics calculations (NAMD). Vibrational modes responsible for the intraband relaxation in (e) $(3 \mathrm{AMP}) \mathrm{Pbl}_{4}$ and (f) (4AMP)Pbl 4 (the vibrational motions of the organic cations are omitted). The DFT and NAMD calculations were performed at the GGA/PBE level with SOC.

both perovskite films show an instantaneous rise in the photobleaching signals that occur at subpicosecond timescales, i.e., $\leq 120$ fs for (4AMP) $\mathrm{PbI}_{4}$ and $120-200 \mathrm{fs}$ for (3AMP) $\mathrm{PbI}_{4}$ at pump fluences from $0.8 \mu \mathrm{J} / \mathrm{cm}^{2}$ to $4.0 \mu \mathrm{J} / \mathrm{cm}^{2}$. This suggests that (i) the excess energy gained by the electrons in 2D DJ perovskites can simultaneously promote the generation of nonequilibrium LOphonons and (ii) the Auger heating effect is almost negligible at these pump fluences. The fitting parameters for the TA kinetics for (3AMP) $\mathrm{PbI}_{4}$ and (4AMP) $\mathrm{PbI}_{4}$ at different pump fluences are given in Supplementary Tables 4 and 5; they show a longer initial decay at high-energy excitations (i.e., $\tau_{1}=0.45-3.50 \mathrm{ps}$ for (3AMP) $\mathrm{PbI}_{4}$ and $\tau_{1}=0.55-1.86 \mathrm{ps}$ for $(4 \mathrm{AMP}) \mathrm{PbI}_{4}$ ) compared to that obtained with low-energy excitations.

To understand the similar rise and initial decays in the TA kinetics as well as the intraband relaxations governed by nonadiabatic coupling among high-energy levels, we performed nonadiabatic molecular dynamics (NAMD) calculations for (3AMP) $\mathrm{PbI}_{4}$ and $(4 \mathrm{AMP}) \mathrm{PbI}_{4}$ with consideration of electronic coherence effects. Based on the Kohn-Sham energies for the electronic levels obtained at the GGA/PBE + SOC level (Fig. 3c, d), the NAMD calculations only involved high-energy conduction bands (i.e., electron cooling process) as the effective masses for the electrons are much smaller than those for the holes (i.e., $0.18 v s$. $0.34 m_{0}$ for (3AMP) $\mathrm{PbI}_{4} ; 0.32$ vs. $0.81 m_{0}$ for (4AMP) $\mathrm{PbI}_{4}$ ). To quantify the intraband relaxations of hot electrons, the population decay curves were fitted using the equation $f(t)=a \cdot \exp \left(-t / \tau_{1}\right)+$ $(1-a) \cdot \exp \left(-\left(t / \tau_{2}\right)^{2}\right)$ and the relaxation times were calculated according to $\tau_{\text {intraband }}=a \cdot \tau_{1}+(1-a) \cdot \tau_{2}{ }^{30}$.

As shown in Fig. $3 c$, d, the electrons in (3AMP) $\mathrm{PbI}_{4}$ undergo a fast relaxation from the initial $\mathrm{CBM}+7(1.52 \mathrm{eV}$ above the conduction band maximum, CBM) to the band edge, and the population of electrons in the initial state rapidly decays to the $\mathrm{CBM}$ with a $\tau_{\text {intraband }}$ of $54 \mathrm{fs}$. For the case of $(4 \mathrm{AMP}) \mathrm{PbI}_{4}$, the population of hot electrons cools down from the initial CBM +4 to the CBM with a relaxation time of $\tau_{\text {intraband }}=57 \mathrm{fs}$. The comparable intraband relaxation times agree well with the rise times of the photobleaching signals of ( $3 \mathrm{AMP}) \mathrm{PbI}_{4}$ and (4AMP) $\mathrm{PbI}_{4}$. The fast intraband relaxation times can be attributed to the direct population transfer from the initial state to the lower states, i.e., to nonvanishing nonadiabatic couplings (NAC; the NAC mappings are shown in Supplementary Fig. 11) among these states, which emerge once spin-orbit coupling is included in the NAMD calculations: $\mathrm{NAC}[<\mathrm{CBM}+7 \mid \mathrm{CBM}+m>] \quad(m=0-$ $6)=16-28 \mathrm{meV}$ for $(3 \mathrm{AMP}) \mathrm{PbI}_{4} ; \mathrm{NAC}[<\mathrm{CBM}+4 \mid \mathrm{CBM}+$ 
$m>$ ] $(m=0-3)=15-27 \mathrm{meV}$ for (4AMP) $\mathrm{PbI}_{4}$. These couplings allow the acceleration of hot-carrier cooling via multiple intraband relaxation channels ${ }^{31}$.

Analysis of the experimental Raman spectra, calculated Raman modes, and spectral densities between two conduction bands (i.e., pair states), as shown in Supplementary Fig. 12, confirms that low-frequency Raman modes (at $\sim 40 \mathrm{~cm}^{-1}$ for (3AMP) $\mathrm{PbI}_{4}$ and $\sim 50 \mathrm{~cm}^{-1}$ for (4AMP) $\mathrm{PbI}_{4}$ ) are responsible for the hot-carrier cooling. In a way similar to the vibrational features found in $3 \mathrm{D}$ perovskite structures ${ }^{16,27}$, the major low-frequency modes can be assigned to the horizontal $\mathrm{Pb}-\mathrm{I}$ stretching modes and vertical $\mathrm{I}-\mathrm{Pb}-\mathrm{I}$ rocking modes of the inorganic layer (see the vibrational vectors for the major modes in Fig. $3 \mathrm{e}, \mathrm{f}$ ). Since the organic spacers in $2 \mathrm{D}$ perovskites are predominantly coupled to the stretching/rocking vibrations of the inorganic layers, these lowfrequency "hybrid phonon" modes can accelerate the hot-carrier cooling processes ${ }^{13}$. Moreover, the hot-carrier cooling in lead halide perovskites is also controlled by electron-phonon coupling (i.e., the interactions between the LO-phonons and electrons $)^{15,32}$, which can be described by the Fröhlich parameter ( $\alpha$, see Supplementary Note 4 ). As given in Supplementary Table 6, 2D DJ perovskites have smaller calculated $\alpha$ values $(2.53$ for (3AMP) $\mathrm{PbI}_{4}$ and 2.29 for $(4 \mathrm{AMP}) \mathrm{PbI}_{4}$ ) than the 2D RP perovskites (3.36 for $(\mathrm{PMA})_{2} \mathrm{PbI}_{4}$ and 4.29 for $\left.(\mathrm{PEA})_{2} \mathrm{PbI}_{4}\right)$, indicating that the electronic transitions among the conduction bands in $2 \mathrm{D} \mathrm{DJ}$ perovskites are less coupled to the vibrations of the inorganic cages. Moreover, (3AMP) $\mathrm{PbI}_{4}$ and (4AMP) $\mathrm{PbI}_{4}$ have similar screened Coulomb interactions among excited charge carriers, as the dielectric constants for the organic cations are almost the same. Although the lifetimes of the intraband relaxations are slightly longer in 2D RP perovskites because of weaker nonadiabatic couplings (Supplementary Fig. 13), the Coulomb interactions are less screened due to the small dielectric constant of the cations (see Supplementary Note 5), leading to a more significant scattering of the hot carriers with optical phonons.

Spin-selective hot carrier cooling processes in 2D DJ perovskites. One way to retard hot-carrier cooling at longer delays in (4AMP) $\mathrm{PbI}_{4}$ is to manipulate the hot carriers via band splitting. To confirm and further understand the role of band splitting in the hot-carrier cooling processes, we performed circular polarized transient absorption (CTA) measurements for (3AMP) $\mathrm{PbI}_{4}$ and (4AMP) $\mathrm{PbI}_{4}$ films. In the CTA measurements, the high-energy excitations were set to be polarized either right-handed circular $\left(\sigma^{+}\right)$or left-handed circular $\left(\sigma^{-}\right)$, and a copolarized (counterpolarized) probe pulse was used to probe the hot carrier relaxation from spin-up and spin-down states (see the scheme for the experimental setup in Supplementary Fig. 14). As illustrated in Fig. $4 a$, spin states with total angular momentum $(\mid+1>$ or $\mid-1>)$ and high-energy levels $(\mid+n>$ or $\mid-n>)$ are generated using the polarized pump pulse. The normalized cocircular and counter circular pump-probe TA spectra of the (3AMP) $\mathrm{PbI}_{4}$ and (4AMP) $\mathrm{PbI}_{4}$ films are shown in Supplementary Figs. 15 and 16, and the corresponding fitting parameters for the kinetics probed at the photobleaching peaks are given in Supplementary Table 7.

Figure $4 \mathrm{~b}, \mathrm{c}$ show the $T_{\mathrm{c}}$ evolution of ( $\left.3 \mathrm{AMP}\right) \mathrm{PbI}_{4}$ and (4AMP) $\mathrm{PbI}_{4}$ following high-energy excitation with a pump fluence of 4.0 $\mu \mathrm{J} / \mathrm{cm}^{2}$. For the case of (3AMP) $\mathrm{PbI}_{4}$, the $T_{c}$ decays are quite similar to those observed in the TA measurements and are not sensitive to the type of circular polarization for the pump and probe. However, (4AMP) $\mathrm{PbI}_{4}$ shows a fast initial decay of $T_{\mathrm{c}}$ and a slower decay of tens of picoseconds when $T_{\mathrm{c}}$ approaches $400 \mathrm{~K}$, especially with the copolarized $\sigma^{+} \sigma^{+}$pump and probe. The extracted $T_{\mathrm{c}}$ lifetimes and the initial decays $\left(\tau_{1}\right)$ for the (4AMP)
$\mathrm{PbI}_{4}$ film are 4-6 ps and 2-3.5 ps, respectively, both of which are much higher than those obtained by the TA measurements with the same pump fluence. Therefore, the Rashba band splitting in (4AMP) $\mathrm{PbI}_{4}$ can induce an additional barrier to spin flipping because $\mid+n>$ and $\mid-n>$ are present in different momentum valleys, as well as reduced spin-phonon scattering of hot electrons from two different spin states. We further performed NAMD calculations by treating the hot electrons from two split conduction bands with consideration of electronic decoherence effects as the high-energy levels of (4AMP) $\mathrm{PbI}_{4}$ are well separated $^{33}$. As shown in Fig. $4 \mathrm{~d}$, the lifetimes of hot electrons in (4AMP) $\mathrm{PbI}_{4}$ with spin-up and spin-down states are $\sim 228 \mathrm{fs}$, which is much longer than that obtained with consideration of coherence effects and agrees well with the rise time ( $\tau_{\text {rise }}=$ 190-210 fs) obtained from the CTA kinetics. However, (3AMP) $\mathrm{PbI}_{4}$ retains a similar rise time ( $\tau_{\text {rise }}=\sim 180-200 \mathrm{fs}$ ) as compared to that obtained from TA kinetics following the same pump fluence $\left(4 \mu \mathrm{J} / \mathrm{cm}^{2}\right)$.

The schematic illustration in Fig. 4e depicts the hot-carrier cooling processes in 2D DJ and RP perovskites. In all cases, the initial cooling processes are mainly governed by the nonequilibrium LO-phonon population (i.e., the scattering of hot electrons with LO-phonons). Then, the hot phonon emission contributes to slow hot-carrier cooling up to tens of picoseconds, especially at a high pump fluence. Moreover, the hot-carrier cooling process can be retarded by Coulomb screening effects, as the macroscopic electric field induced by the out-of-phase displacements of the atoms is weakened by Coulomb screening. For (4AMP) $\mathrm{PbI}_{4}$, the Rashba band splitting means that the hotcarrier cooling is not only dependent on the interactions between hot electrons and LO-phonons but also affected by spindependent scattering of hot electrons ${ }^{34}$. The D'yakonov-Perel' (DP) mechanism dominates in (4AMP) $\mathrm{PbI}_{4}$ in which there is no inversion symmetry, and the Rashba effect induces spin precession randomized by scattering due to the SOC-induced internal effective magnetic field ${ }^{35}$. In addition, strong spinphonon scattering can decrease the hot-carrier lifetime, as it gives rise to faster spin randomization and flip. To minimize spindependent scattering, hot carriers with spin-up or spin-down states can be manipulated through circular copolarized light excitation; in that instance, the hot-carrier temperature approaches $400 \mathrm{~K}$ and has a longer lifetime.

In summary, we have demonstrated the significant influence of Rashba band splitting on the hot-carrier cooling dynamics in $2 \mathrm{D}$ Dion-Jacobson hybrid perovskites. Electronic band structure calculations and measurements of the temperature-dependent PL spectra and PL decay were used to verify the presence of Rashba band splitting in (4AMP) $\mathrm{PbI}_{4}$, which originates from the symmetry breaking by in-plane equatorial distortions. The scattering between hot electrons and LO-phonons governs the initial fast cooling in both (3AMP) $\mathrm{PbI}_{4}$ and (4AMP) $\mathrm{PbI}_{4}$. The spin-dependent scattering of hot electrons generated by the Rashba effect can accelerate hot-carrier cooling in (4AMP) $\mathrm{PbI}_{4}$. Importantly, the cooling process can be slowed down by controlling the spin states of the hot carriers with circular copolarized excitation. Our findings highlight the importance of Rashba band splitting in two-dimensional perovskites as a powerful tool to control hot-carrier cooling dynamics, which can be exploited to design and synthesize $2 \mathrm{D}$ materials with promising optoelectronic and spintronic applications.

\section{Methods}

Materials. Lead(II) oxide ( $\mathrm{PbO}>99 \%)$, lead(II) iodide $\left(\mathrm{PbI}_{2}>99 \%\right)$, 3-(aminomethyl)piperidine (3AMP), 4-(aminomethyl)piperidine (4AMP), benzylamine, hypophosphorous acid $\left(\mathrm{H}_{3} \mathrm{PO}_{2}\right), \mathrm{N}, \mathrm{N}$-dimethylformamide (DMF), dimethyl sulfoxide (DMSO) and diethyl ether (DEE) were purchased from Sigma Aldrich and 

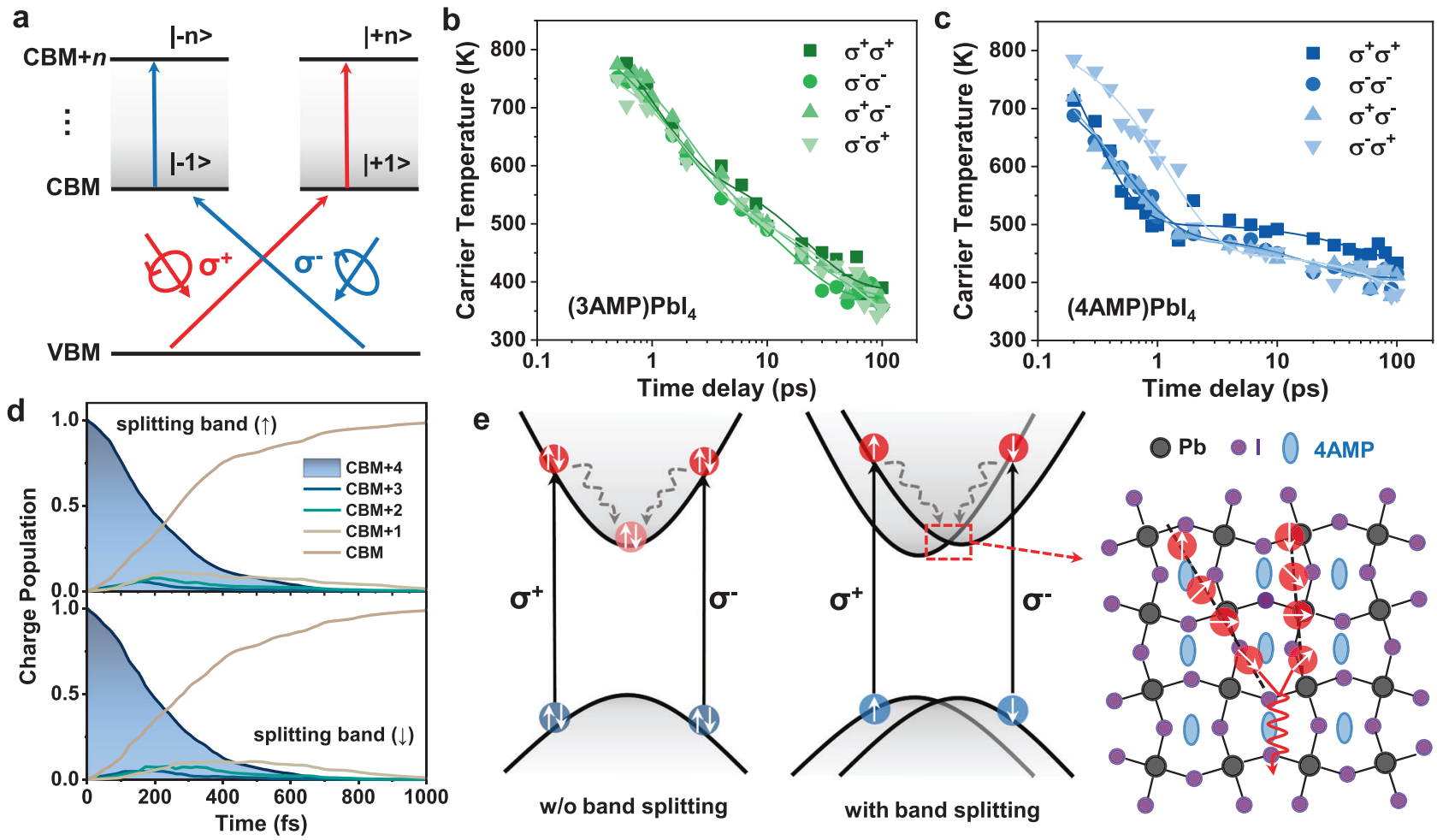

Fig. 4 Spin-selective hot carrier relaxation of 2D Dion-Jacobson perovskites. a Scheme for the optical selection for the circular polarized TA measurements of 2D DJ perovskite films. Right (left) circular polarized light $\sigma^{+}\left(\sigma^{-}\right)$couples to the electronic transition from the valence bands to highlevel conduction bands. Extracted hot-carrier temperature as a function of delay time of (b) (3AMP) $\mathrm{Pbl}_{4}$ and (c) (4AMP) $\mathrm{Pbl}_{4}$ obtained from cocircular $\left(\sigma^{+} \sigma^{+}\right.$and $\left.\sigma^{-} \sigma^{-}\right)$and counter circular $\left(\sigma^{+} \sigma^{-}\right.$and $\left.\sigma^{-} \sigma^{+}\right)$polarized pump-probe TA spectra. $\mathbf{d}$ Time evolution of hot-electron relaxation starting from the two split bands for (3AMP) $\mathrm{Pbl}_{4}$ calculated with consideration of spin-orbit coupling and decoherence effects. e Schematic illustration of the hot-electron relaxation processes in 2D DJ perovskites without and with band splitting and of the spin-flip/precession and scattering processes in (4AMP)Pbl ${ }_{4}$.

used without further purification. Phenylethylammonium iodide (PEAI) was purchased from GreatCell Solar, and hydroiodic acid (HI, 55-58\%) was obtained from Alfa Aesar.

Synthesis of 2D perovskite single crystals. For the (3AMP) $\mathrm{PbI}_{4}$ and (4AMP) $\mathrm{PbI}_{4}$ single crystals, $1 \mathrm{mmol}$ of $\mathrm{PbO}(223.2 \mathrm{mg})$ was dissolved in $4 \mathrm{~mL}$ of $\mathrm{HI}$ solution under boiling conditions for $5 \mathrm{~min}$. In a separate vial, $1 \mathrm{mmol}$ of $3 \mathrm{AMP}$ (114.0 mg) was dissolved in $0.5 \mathrm{~mL}$ of $\mathrm{H}_{3} \mathrm{PO}_{2}$. Then, under boiling conditions, $\mathrm{H}_{3} \mathrm{PO}_{2}$ neutralized 3AMP solution was added to the $\mathrm{PbO}$-containing vial. The solution was allowed to boil for another $5 \mathrm{~min}$ and then slowly cooled to room temperature at a cooling rate of $3{ }^{\circ} \mathrm{C} /$ hour. The $\left(4 \mathrm{AMP} \mathrm{PbI}_{4}\right.$ crystals were prepared using a similar method. For the (PMA) $)_{2} \mathrm{PbI}_{4}$ and $(\mathrm{PEA})_{2} \mathrm{PbI}_{4}$ single crystals, $\mathrm{PbI}_{2}$ was used as the $\mathrm{Pb}$ precursor, and the synthesis methods have been reported in our previous studies 24,36 .

Fabrication of 2D perovskite thin films. The solutions of single crystals were prepared in $1 \mathrm{~mL}$ of mixed solvent (DMF:DMSO $=80: 20$ ) to reach a concentration of $100 \mathrm{mg} / \mathrm{mL}$. To fabricate the thin films, $100 \mu \mathrm{L}$ of single crystal solution was pipetted onto the glass substrate and spin-coated at 2000 RPM for $60 \mathrm{sec}$. After $40 \mathrm{sec}, 700 \mu \mathrm{L}$ of DEE was added as an antisolvent. Then, the (3AMP) $\mathrm{PbI}_{4}$ and (4AMP) $\mathrm{PbI}_{4}$ films were annealed at $140{ }^{\circ} \mathrm{C}$ for $5 \mathrm{~min}$, and the (PMA) ${ }_{2} \mathrm{PbI}_{4}$ and (PEA) ${ }_{2} \mathrm{PbI}_{4}$ films were annealed at $80^{\circ} \mathrm{C}$ for $5 \mathrm{~min}$. The thicknesses of $2 \mathrm{D} \mathrm{DJ}$ perovskite films were $\sim 300 \mathrm{~nm}$ as measured via the thickness profiler; the root mean square roughness $\left(\sigma_{\mathrm{RMS}}\right)$ was $2.6 \mathrm{~nm}$ for $(3 \mathrm{AMP}) \mathrm{PbI}_{4}$ and $8.7 \mathrm{~nm}$ for (4AMP) $\mathrm{PbI}_{4}$ (see Supplementary Fig. 2)

X-ray diffraction measurements. X-ray diffraction patterns of the (3AMP) $\mathrm{PbI}_{4}$ and (4AMP)PbI ${ }_{4}$ films were recorded using a Bruker D8 Advance $(40 \mathrm{kV}, 40 \mathrm{~mA})$ with $\mathrm{CuK}_{\alpha 1}$ radiation $(\lambda=1.5406 \AA)$ operating at a step size of $0.02^{\circ}$ and a speed of $0.4 \mathrm{sec} / \mathrm{step}$ at room temperature.

Steady-state absorption measurements. Absorption spectra of the (3AMP) $\mathrm{PbI}_{4}$ (4AMP) $\mathrm{PbI}_{4},(\mathrm{PMA})_{2} \mathrm{PbI}_{4}$ and $(\mathrm{PEA})_{2} \mathrm{PbI}_{4}$ films were recorded in absorbance mode using a LAMBDA 1050 (Perkin Elmer).
Raman measurements. Raman measurements were performed using a Witec Apyron instrument equipped with a 1064-nm laser and a Zeiss EC

Epiplan-Neofluar $100 \times / 0.9$ NA Objective lens. The signal integration time was set at $45 \mathrm{~s}$.

Temperature-dependent PL measurements. Temperature-dependent PL measurements were carried out for the (3AMP)PbI ${ }_{4}$ and (4AMP) $\mathrm{PbI}_{4}$ films by using a $488 \mathrm{~nm} \mathrm{Ar}+$ laser. The signals were detected by Andor Shamrock spectrograph attached to Andor Newton CCD camera. Cryogenic measurements were carried out in a closed-cycle cryostat under vacuum with temperatures ranging between $5 \mathrm{~K}$ and $300 \mathrm{~K}$.

Time-resolved PL measurements. Time-resolved PL measurements were performed using the time-correlated single-photon counting technique (TCSPC). The different excitation wavelengths used for the $2 \mathrm{D}$ perovskite films were tuned using a parametric optical amplifier (Newport, Spectra Physics) that was pumped with an ultrafast Ti:sapphire amplifier ( $800 \mathrm{~nm}, 100 \mathrm{fs}, 1 \mathrm{kHz}$, Astrella, Coherent). The energy at each excitation wavelength was modulated by a pair of variable neutral density filters (Thorlabs). The excitation beam entered a Halcyone setup (Ultrafast Systems) where it was focused onto the sample, and the resulting photoluminescence was collected and recollimated by using a pair of parabolic mirrors, passed through longpass filters $(450 \mathrm{~nm}, 550 \mathrm{~nm}$, Newport), and finally focused onto an optical fiber directed towards the monochromator and detector. To ensure that less than $1 \%$ of the excitation events resulted in photon detection, an additional variable ND filter was used before the optical fiber. The 2D perovskite films were measured in the reflection arrangement of the system, and the obtained TCSPC histograms were fitted using the Lavenberg-Marquart algorithm implemented in Ultrafast Systems software. The overall setup has a time resolution of $110 \mathrm{ps}$. The detection fiber optics are coupled to a multichannel spectrometer with a CMOS sensor that has a $1.5-\mathrm{nm}$ intrinsic resolution with maximum spectral acquisition rate of $9500 \mathrm{spectra} / \mathrm{s}$.

Femtosecond transient absorption measurements. Femtosecond transient absorption measurements were performed for the 2D perovskite films by using a multipass amplified Ti:sapphire laser $(800 \mathrm{~nm}$ laser pulses with a $7 \mathrm{~mJ} /$ pulse; pulse width of $\sim 100 \mathrm{fs}$ with a repetition rate of $1 \mathrm{kHz}$, Astrella from Coherent) in conjunction with Helios spectrometers. Different excitation pump pulses (see the 
summary in Supplementary Table 2) were generated after passing through a fraction of the $800 \mathrm{~nm}$ beam into a spectrally tunable $(240-2600 \mathrm{~nm})$ optical parametric amplifier (Newport Spectra-Physics). The probe pulses (UV-Visible and NIR wavelength continuum, white light) were generated by passing another fraction of the $800 \mathrm{~nm}$ pulses through a $2 \mathrm{~mm}$ thick calcium fluoride $\left(\mathrm{CaF}_{2}\right)$ crystal. The absorption change $(\Delta \mathrm{A})$ was measured with respect to the time delay and wavelength. Circular polarized TA measurements were performed using the same setup with an adjustment (see Supplementary Fig. 14). A half waveplate $(\lambda / 2)$ and a fixed circular polarizer were set to control the pump polarization. The probe pulses were detected either parallel or perpendicular to the pump pulses, which was controlled by a variable polarizer.

Density functional theory (DFT) calculations. DFT calculations were carried out for $2 \mathrm{D}$ perovskites, $(\mathrm{PMA})_{2} \mathrm{PbI}_{4},(\mathrm{PEA})_{2} \mathrm{PbI}_{4},(3 \mathrm{AMP}) \mathrm{PbI}_{4}$, and $(4 \mathrm{AMP}) \mathrm{PbI}_{4}$, using the projector-augmented wave (PAW) method as implemented in the VASP $\operatorname{code}^{37,38}$. The generalized gradient approximation (GGA) with the

Perdew-Burke-Ernzerhof (PBE) exchange-correlation functional was used. A uniform k-mesh grid of $4 \times 4 \times 2$ was used for $(\mathrm{PMA})_{2} \mathrm{PbI}_{4},(\mathrm{PEA})_{2} \mathrm{PbI}_{4}$, and (4AMP) $\mathrm{PbI}_{4}$, and $4 \times 2 \times 2$ was used for ( $3 \mathrm{AMP} \mathrm{PbI}_{4}$. The plane-wave basis set cutoffs for the wavefunctions were set at $450 \mathrm{eV}$. Starting from the experimental lattice parameters (CCDC 1831521 for (3AMP) $\mathrm{PbI}_{4}$ and CCDC 1831525 for (4AMP) $\mathrm{PbI}_{4}{ }^{22}$ ), the atomic positions for all $2 \mathrm{D}$ perovskites were fully relaxed by relaxing both the cell parameters and the atomic positions until the supercells had forces of less than $0.01 \mathrm{eV} / \AA \AA$ on each atom. The Heyd-Scuseria-Ernzerhof hybrid functional (HSE06), including spin-orbit coupling, was used to calculate the electronic band structures (Fig. 1c, d)

The Raman and far-infrared vibrational mode positions and intensities of the 2D perovskites were calculated by using the Phonon code as implemented in the Quantum ESPRESSO (QE) package ${ }^{39,40}$. The local density approximation (LDA) exchange-correlation functional with norm-conserving pseudopotentials was used, and the plane-wave basis set cutoffs for the wavefunctions were set at 90 Ry with a self-consistency threshold of $10^{-14} \mathrm{Ry}$. The SOC was not included in the Raman calculations, as it plays a less significant role than the geometry in the description of the vibrational properties of heavy metal-based perovskite systems.

Nonadiabatic molecular dynamics (NAMD) calculations. The crystal structures of the 2D perovskites were further optimized at the GGA/PBE level using the PWSCF code as implemented in the QE package ${ }^{39}$. Ultrasoft pseudopotentials were used considering spin-orbit coupling. Uniform Brillouin zone grids of $4 \times 4 \times 2 \mathrm{k}$ mesh for $(\mathrm{PMA})_{2} \mathrm{PbI}_{4},(\mathrm{PEA})_{2} \mathrm{PbI}_{4}$, and $(4 \mathrm{AMP}) \mathrm{PbI}_{4}$ and $4 \times 2 \times 2$ for $(3 \mathrm{AMP}) \mathrm{PbI}_{4}$ were employed, and the plane-wave basis set cutoffs for the wavefunctions and charge density were set at $40 \mathrm{Ry}$ and $300 \mathrm{Ry}$, respectively. The optimized crystal structures of the $2 \mathrm{D}$ perovskites were considered as the starting point for calculating the ground-state molecular dynamics trajectories, where a $1000 \mathrm{fs}$ trajectory for the system was obtained. The Andersen thermostat was used to control the temperature of the system at $300 \mathrm{~K}$. The initial $1000 \mathrm{fs}$ for the trajectories with a $1 \mathrm{fs}$ time step were obtained for nuclear thermalization, and the subsequent $2000 \mathrm{fs}$ were used for the NAMD calculations.

NAMD calculations for the 2D perovskites were carried out using the PYXAID2 $\operatorname{code}^{33,41}$. The fewest-switches surface hopping algorithm ${ }^{42}$ implemented within time-dependent DFT was used to investigate the hybrid perovskite systems ${ }^{43-45}$. Starting from the time-dependent Schrödinger equation $i \hbar \frac{\partial \Psi_{n}(\mathbf{r}, t)}{\partial t}=$

$H(\mathbf{r}, \mathbf{R}, t) \Psi_{n}(\mathbf{r}, \mathrm{t})$ and Kohn-Sham orbitals $\Psi_{n}(\mathbf{r}, \mathbf{t})=\sum_{k} C_{k}^{n}(t) \stackrel{\partial t}{\Phi}(\mathbf{r} ; \mathbf{R})$, the hot carrier relaxations were investigated by calculating the average energy and population of charge carriers from several excited states. The probability of transition between adiabatic states $i$ and $j$ can be calculated using the wavefunction expansion coefficients and coupling, defined as $d_{i j}=-i \hbar\left\langle\Phi_{i}\left|\frac{\partial}{\partial t}\right| \Phi_{j}\right\rangle$. Detailed descriptions for the NAMD theory can be found in Refs. ${ }^{33,41}$. A total of 1500 geometries were randomly selected from the adiabatic trajectories and used as initial conditions in the NAMD calculations.

\section{Data availability}

The data that support the findings of this study are available from the corresponding author upon reasonable request.

\section{Code availability}

Vienna ab initio simulation package (VASP) for the periodic DFT calculations is available at https://www.vasp.at/. Quantum Espresso code for the phonon calculations is freely available at https://www.quantum-espresso.org/. PYXAID code for the nonadiabatic molecular dynamics calculations is available at https://github.com/ quantum-dynamics-hub/pyxaid2.

Received: 9 March 2021; Accepted: 3 June 2021; Published online: 28 June 2021

\section{References}

1. Blancon, J. C., Even, J., Stoumpos, C. C., Kanatzidis, M. G. \& Mohite, A. D. Semiconductor physics of organic-inorganic 2D halide perovskites. Nat. Nanotechnol. 15, 969-985 (2020).

2. Gong, X. W. et al. Electron-phonon interaction in efficient perovskite blue emitters. Nat. Mater. 17, 550-556 (2018).

3. Tsai, H. H. et al. A sensitive and robust thin-film X-ray detector using $2 \mathrm{D}$ layered perovskite diodes. Sci. Adv. 6, eaay0815 (2020).

4. Blancon, J. C. et al. Scaling law for excitons in $2 \mathrm{D}$ perovskite quantum wells. Nat. Commun. 9, 2254 (2018).

5. Giovanni, D. et al. Tunable room-temperature spin-selective optical Stark effect in solution-processed layered halide perovskites. Sci. Adv. 2, e1600477 (2016).

6. Giovanni, D. et al. Coherent spin and quasiparticle dynamics in solutionprocessed layered 2D lead halide perovskites. Adv. Sci. 5, 1800664 (2018).

7. Guo, P. J. et al. Cross-plane coherent acoustic phonons in two-dimensional organic-inorganic hybrid perovskites. Nat. Commun. 9, 2019 (2018).

8. Maity, P. et al. Layer-dependent coherent acoustic phonons in twodimensional ruddlesden-popper perovskite crystals. J. Phys. Chem. Lett. 10, 5259-5264 (2019).

9. Zhai, Y. X. et al. Giant Rashba splitting in 2D organic-inorganic halide perovskites measured by transient spectroscopies. Sci. Adv. 3, e1700704 (2017).

10. Yin, J. et al. Layer-dependent Rashba band splitting in 2D hybrid perovskites. Chem. Mater. 30, 8538-8545 (2018).

11. Tan, L. Z. et al. Shift current bulk photovoltaic effect in polar materials-hybrid and oxide perovskites and beyond. NPJ Comput. Mater. 2, 16026 (2016).

12. Yuan, Y. B. et al. Anomalous photovoltaic effect in organic-inorganic hybrid perovskite solar cells. Sci. Adv. 3, e1602164 (2017).

13. Yin, J. et al. Tuning hot carrier cooling dynamics by dielectric confinement in two-dimensional hybrid perovskite crystals. ACS Nano 13, 12621-12629 (2019).

14. Wang, T. et al. Protecting hot carriers by tuning hybrid perovskite structures with alkali cations. Sci. Adv. 6, eabb1336 (2020).

15. Yang, Y. et al. Observation of a hot-phonon bottleneck in lead-iodide perovskites. Nat. Photonics 10, 53-59 (2016).

16. Fu, J. H. et al. Hot carrier cooling mechanisms in halide perovskites. Nat. Commun. 8, 1300 (2017).

17. Joshi, P. P., Maehrlein, S. F. \& Zhu, X. Y. Dynamic screening and slow cooling of hot carriers in lead halide perovskites. Adv. Mater. 31, 1803054 (2019).

18. Miyata, K. et al. Large polarons in lead halide perovskites. Sci. Adv. 3, e1701217 (2017).

19. Miyata, K., Atallah, T. L. \& Zhu, X. Y. Lead halide perovskites: Crystal-liquid duality, phonon glass electron crystals, and large polaron formation. Sci. Adv. 3, e1701469 (2017).

20. Konig, D. et al. Hot carrier solar cells: principles, materials and design. Phys. E 42, 2862-2866 (2010)

21. Park, I. H. et al. Ferroelectricity and rashba effect in a two-dimensional Dion-Jacobson hybrid organic-inorganic perovskite. J. Am. Chem. Soc. 141, 15972-15976 (2019)

22. Mao, L. L. et al. Hybrid Dion-Jacobson 2D lead iodide perovskites. J. Am. Chem. Soc. 140, 3775-3783 (2018).

23. Mao, L. L. et al. Organic cation alloying on intralayer A and interlayer A' sites in $2 \mathrm{D}$ hybrid Dion-Jacobson lead bromide perovskites (A')(A) $\mathrm{Pb}_{2} \mathrm{Br}_{7} . J . A m$. Chem. Soc. 142, 8342-8351 (2020).

24. Yin, J. et al. Modulation of broadband emissions in two-dimensional $<100>$ oriented Ruddlesden-Popper hybrid perovskites. ACS Energy Lett. 5, 2149-2155 (2020)

25. Fang, H. H., Adjokatse, S., Shao, S. Y., Even, J. \& Loi, M. A. Long-lived hotcarrier light emission and large blue shift in formamidinium tin triiodide perovskites. Nat. Commun. 9, 243 (2018).

26. Price, M. B. et al. Hot-carrier cooling and photoinduced refractive index changes in organic-inorganic lead halide perovskites. Nat. Commun. 6, 8420 (2015).

27. Li, M. J. et al. Slow cooling and highly efficient extraction of hot carriers in colloidal perovskite nanocrystals. Nat. Commun. 8, 14350 (2017).

28. Klimov, V. I. \& McBranch, D. W. Femtosecond 1p-to-1s electron relaxation in strongly confined semiconductor nanocrystals. Phys. Rev. Lett. 80, 4028-4031 (1998).

29. Yu, P. R., Nedeljkovic, J. M., Ahrenkiel, P. A., Ellingson, R. J. \& Nozik, A. J. Size dependent femtosecond electron cooling dynamics in cdse quantum rods. Nano Lett. 4, 1089-1092 (2004).

30. Madlet, M. E. et al. Cation effect on hot carrier cooling in halide perovskite materials. J. Phys. Chem. Lett. 8, 4439-4445 (2017).

31. Li, W., Zhou, L. J., Prezhdo, O. V. \& Akimov, A. V. Spin-orbit interactions greatly accelerate nonradiative dynamics in lead halide perovskites. ACS Energy Lett. 3, 2159-2166 (2018). 
32. Yang, J. F. et al. Acoustic-optical phonon up-conversion and hot-phonon bottleneck in lead-halide perovskites. Nat. Commun. 8, 14120 (2017)

33. Akimov, A. V. \& Prezhdo, O. V. Advanced capabilities of the pyxaid program: Integration schemes, decoherenc effects, multiexcitonic states, and fieldmatter interaction. J. Chem. Theory Comput. 10, 789-804 (2014).

34. Todd, S. B. et al. Detection of Rashba spin splitting in 2D organic-inorganic perovskite via precessional carrier spin relaxation. APL Mater. 7, 081116 (2019).

35. Dyakonov, M. I. \& Perel, V. I. Spin relaxation of conduction electrons in noncentrosymmetric semiconductors. Sov. Phys. Solid State 13, 3023-3026 (1972).

36. Schmitt, T. et al. Control of crystal symmetry breaking with halogensubstituted benzylammonium in layered hybrid metal-halide perovskites. J. Am. Chem. Soc. 142, 5060-5067 (2020).

37. Kresse, G. \& Hafner, J. Ab-initio molecular-dynamics for open-shell transition-metals. Phys. Rev. B 48, 13115-13118 (1993).

38. Kresse, G. \& Furthmuller, J. Efficient iterative schemes for ab initio total-energy calculations using a plane-wave basis set. Phys. Rev. B 54, 11169-11186 (1996).

39. Giannozzi, P. et al. Quantum Espresso: a modular and open-source software project for quantum simulations of materials. J. Phys. Condens. Matter 21, 395502 (2009).

40. Giannozzi, P. et al. Advanced capabilities for materials modelling with quantum espresso. J. Phys. Condens. Matter 29, 465901 (2017).

41. Akimov, A. V. \& Prezhdo, O. V. The PYXAID program for non-adiabatic molecular dynamics in condensed matter systems. J. Chem. Theory Comput. 9, 4959-4972 (2013).

42. Tully, J. C. Molecular-dynamics with electronic-transitions. J. Chem. Phys. 93, 1061-1071 (1990).

43. Liu, J. \& Prezhdo, O. V. Chlorine doping reduces electron-hole recombination in lead iodide perovskites: time-domain ab initio analysis. J. Phys. Chem. Lett. 6, 4463-4469 (2015).

44. Long, R., Liu, J. \& Prezhdo, O. V. Unravelling the effects of grain boundary and chemical doping on electron-hole recombination in $\mathrm{CH}_{3} \mathrm{NH}_{3} \mathrm{PbI}_{3}$ perovskite by time-domain atomistic simulation. J. Am. Chem. Soc. 138, 3884-3890 (2016).

45. Long, R., Fang, W. H. \& Prezhdo, O. V. Moderate humidity delays electron-hole recombination in hybrid organic-inorganic perovskites: Time-domain ab initio simulations rationalize experiments. J. Phys. Chem. Lett. 7, 3215-3222 (2016).

\section{Acknowledgements}

This work was supported by the King Abdullah University of Science and Technology (KAUST) and the College of Science of the University of Arizona. J.Y., J.L.B., and O.F.M. acknowledge the Supercomputing Laboratory at KAUST for their computational and storage resources, as well as their gracious assistance.

\section{Author contributions}

J.Y. conceived the idea and designed the theoretical work. J.Y., O.M.B. and O.F.M. crafted the experimental plan and directed the research. J.Y. performed all the theoretical calculations. J.Y., J.L.B., and O.F.M. analyzed the theoretical results. R.N. prepared the film samples and measured the PL and absorption spectra. P.M. performed the transient absorption spectra measurements. L.G.A. performed the time-resolved PL measurements. D.A. and I.S.R. performed the temperature-dependent PL measurements. J.Y., J.L.B., and O.F.M. wrote the manuscript. All authors discussed and commented on the manuscript.

\section{Competing interests}

The authors declare no competing interests.

\section{Additional information}

Supplementary information The online version contains supplementary material available at https://doi.org/10.1038/s41467-021-24258-7.

Correspondence and requests for materials should be addressed to J.-L.B., O.M.B. or O.F.M.

Peer review information Nature Communications thanks Kian Ping Loh, Xiaoming Wen and the other, anonymous, reviewer(s) for their contribution to the peer review of this work.

Reprints and permission information is available at http://www.nature.com/reprints

Publisher's note Springer Nature remains neutral with regard to jurisdictional claims in published maps and institutional affiliations.

\begin{abstract}
(c) (i) Open Access This article is licensed under a Creative Commons c. Attribution 4.0 International License, which permits use, sharing, adaptation, distribution and reproduction in any medium or format, as long as you give appropriate credit to the original author(s) and the source, provide a link to the Creative Commons license, and indicate if changes were made. The images or other third party material in this article are included in the article's Creative Commons license, unless indicated otherwise in a credit line to the material. If material is not included in the article's Creative Commons license and your intended use is not permitted by statutory regulation or exceeds the permitted use, you will need to obtain permission directly from the copyright holder. To view a copy of this license, visit http://creativecommons.org/ licenses/by/4.0/.
\end{abstract}

(C) The Author(s) 2021 